\title{
Face Representation Using Combined Method of Gabor Filters, Wavelet Transformation and DCV and Recognition Using RBF
}

\author{
Kathirvalavakumar Thangairulappan ${ }^{1 *}$, Jebakumari Beulah Vasanthi Jeyasingh ${ }^{2}$ \\ ${ }^{1}$ Department of Computer Science, VHNSN College, Virudhunagar, India; ${ }^{2}$ Department of Computer Applications, ANJA College, \\ Sivakasi, India. \\ Email: "kathirvalavakumar@yahoo.com, jebaarul07@yahoo.com
}

Received April 27 $7^{\text {th }}, 2012$; revised July $19^{\text {th }}, 2012$; accepted July $26^{\text {th }}, 2012$

\begin{abstract}
An efficient face representation is a vital step for a successful face recognition system. Gabor features are known to be effective for face recognition. The Gabor features extracted by Gabor filters have large dimensionality. The feature of wavelet transformation is feature reduction. Hence, the large dimensional Gabor features are reduced by wavelet transformation. The discriminative common vectors are obtained using the within-class scatter matrix method to get a feature representation of face images with enhanced discrimination and are classified using radial basis function network. The proposed system is validated using three face databases such as ORL, The Japanese Female Facial Expression (JAFFE) and Essex Face database. Experimental results show that the proposed method reduces the number of features, minimizes the computational complexity and yielded the better recognition rates.
\end{abstract}

Keywords: Feature Extraction; Gabor Wavelet; Wavelet Transformation; Discriminative Common Vector; Radial Basis Function Neural Network

\section{Introduction}

Face recognition is one of the most dynamic research areas in the study of pattern recognition and computer vision. A good face recognition methodology should consider representation as well as classification issues [1]. In the literature of face recognition, there are various face representation methods based on global features, including a great number of subspace-based methods and some spatial-frequency techniques. Subspace-based methods, such as principal component analysis (PCA) [2], Fisher's linear discriminant (FLD) [3] and independent component analysis (ICA) [4], have been widely recognized as the dominant and successful face representation methods.

The characteristics of the Gabor wavelets, especially for frequency and orientation representations, are similar to those of the human visual system, and they have been found to be appropriate for texture representation and discrimination. Yi-Chun Lee and Chin-Hsing Chen [5] have proposed feature extraction for face recognition based on Gabor filters and two-dimensional locality preserving projections. Gabor wavelets have been successfully and widely applied to face recognition [6,7], face

\footnotetext{
"Corresponding author.
}

detection [8], texture segmentation [9], handwritten numerals recognition [10] and fingerprint recognition [11]. Chengjun Liu and Harry Wechsler [1] have applied the Enhanced Fisher linear discriminant Model (EFM) to an augmented Gabor feature vector derived from the Gabor wavelet representation of face images to obtain a lowdimensional feature representation with enhanced discrimination power. In the Independent Gabor Features (IGF) method, they have first derived a Gabor feature vector from a set of down sampled Gabor wavelet representation of face images, then reduce the dimensionality of the vector by means of Principal Component Analysis (PCA), and finally defined the independent Gabor features based on the Independent Component Analysis [12]. Arindam Kar et al. [13] have presented a technique by which high intensity feature vectors extracted from the Gabor wavelet transformation of frontal face images, is combined together with Independent Component Analysis (ICA) for enhanced face recognition. Shen et al. [14] have presented a frame work based on a combination of Gabor wavelets and General Discriminant Analysis for face identification and verification. Wing-Pong Choi et al. [15] have proposed a simplified version of Gabor wavelets (SGWs) and an efficient algorithm for extracting the features based on an integral image. 
Cevikalp et al. [16] have proposed a face recognition method called the Discriminative Common Vector (D$\mathrm{CV}$ ), in which within-class scatter matrix of the sample is used to obtain the discriminative common vectors. Among the so many popular methods for face recognition, the wavelet transform is used almost as widely as the subspace method [17]. Its ability to capture localized time-frequency information of image motivates its use for feature extraction. An approach based on a combination of the discrete wavelet transform and the Gabor filter, is implemented in a breast cancer screening system. The two-dimensional discrete wavelet transform is employed to process the mammogram and obtain its HH high frequency sub-band image. Then, a Gabor filter bank is applied to the latter at different frequencies and spatial orientations to obtain new Gabor images [18].

Neural networks have been widely used for classification and recognition tasks. Radial Basis Function Neural Network is a special type of artificial neural network suitable for classification, time-series forecasting and so on. The mostly adopted network topology is radial basis function neural network (RBFNN) due to a number of advantages compared with other types of ANNs, such as better prediction capabilities, simpler network structures, and faster learning process [19]. A novel face recognition approach based on kernel discriminative common vectors (KDCV) and RBF network is proposed [20]. In this, kernel DCV (KDCV) algorithm is employed to generate $\mathrm{DCV}$ and is used as the hidden-layer units of the RBF network for recognizing the patterns. Balasubramanian et al. [21] have presented a method for automatic real time face and mouth in video sequences recognition using radial basis function neural networks (RBFNN).

In this paper, a face recognition system using the combination of Gabor filter, wavelet transformation and DCV has been proposed for feature extraction and then radial basis function is used to recognize the extracted features. The rest of the paper is structured as follows: the next section describes feature extraction using Gabor wavelet, wavelet transformation and the discriminative common vector method. Section 3 presents the proposed recognition process using radial basis function network. Section 4 describes the data set and experiment results along with discussions.

\section{Feature Extraction}

Feature extraction, in the sense of linear or nonlinear transform of the data with subsequent feature selection is commonly used for reducing the dimensionality of the patterns. In the proposed work, Gabor filters and the wavelet transformation are applied on the input patterns to extract the important features and reduce the dimension and then discriminative common vectors are ob- tained using within-class scatter matrix.

\subsection{Gabor Wavelet Representation of Faces}

In this work, Gabor features are used to represent the face images. Gabor filters are defined as follows. In the spatial domain, a 2D Gabor filter is a Gaussian kernel function modulated by a sinusoidal plane wave $[22,23]$ is as follows:

$$
\begin{aligned}
& \varphi_{\Pi(f, \theta, \gamma, \eta)}(x, y) \\
& =\frac{f^{2}}{\pi \gamma \eta} \exp \left(-\left(\alpha^{2} x^{\prime 2}+\beta^{2} y^{\prime 2}\right)\right) \exp \left(j 2 \pi f x^{\prime}\right), \\
& x^{\prime}=x \cos \theta+y \sin \theta \\
& y^{\prime}=-x \sin \theta+y \cos \theta
\end{aligned}
$$

where $f$ is the central frequency of the sinusoidal plane wave, $\theta$ is the anti-clockwise rotation of the Gaussian and the plane wave, $\alpha$ is the sharpness of the Gaussian along the major axis parallel to the wave, and $\beta$ is the sharpness of the Gaussian minor axis perpendicular to the wave. $\gamma=\frac{f}{\alpha}$ and $\eta=\frac{f}{\beta}$ are defined to keep the ratio between frequency and sharpness constant. To extract features from a face image, a set of Gabor filters with different frequencies and orientations are required as,

$$
\begin{gathered}
\varphi_{u, v}=\varphi_{\Pi(f, \theta, \gamma, \eta)}(x, y), f_{u}=f_{\max } / \sqrt{2}^{u}, \theta_{v}=\frac{v}{8} \pi \\
u=0, \cdots, U-1, v=0, \cdots, V-1
\end{gathered}
$$

where $f_{\max }$ highest peak frequency, $U$ and $V$ are the number of scales and orientations respectively. An image can be represented by the Gabor wavelet transform with the description of both the spatial frequency structure and spatial relations. The number of Gabor filters to use is the first issue to deal with for feature extraction from images. This depends on the application. Normally 40 filters such as 5 scales and 8 orientations as shown in Figure $\mathbf{1}$ are used for face recognition. In this work, Gabor filters designed with 5 scales and 8 orientations are used for feature extraction.

Image features can be extracted by convolving the input image with Gabor filters. The Gabor representation of a face image $I(\boldsymbol{x})$ can be obtained by convolving the image with the Gabor filters as defined by

$$
G_{u, v}(\boldsymbol{x})=\left(I * \varphi_{(u, v)}\right)(\boldsymbol{x})
$$

where $G_{u, v}(x)$ denotes the convolution result corresponding to the Gabor filter at orientation $u$ and scale $v$. As a result, image $I(\boldsymbol{x})$ can be represented by a set of Gabor wavelet coefficients 
$G_{u, v}(\boldsymbol{x}), u=0, \cdots, 4 ; v=0, \cdots, 7$. Convolving the input image with the 40 Gabor filters with 5 different scales and 8 orientations results in the Gabor feature set. An input face image and the Gabor feature representations are shown in the Figure 2.

\subsection{Wavelet Transform}

Wavelet transformation results in strong representations with regard to lighting changes and be capable of capturing substantial facial features. A wavelet transform is created by passing the image through a series of filter bank stages. The filtered outputs are then down sampled by a factor of 2 in the horizontal direction. Each of these signals is then filtered by an identical filter pair in the vertical direction. The decomposition of the image into 4

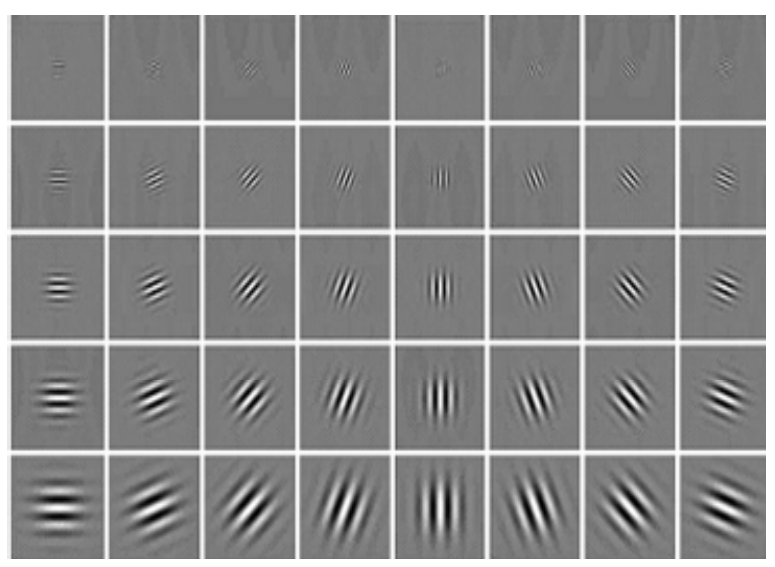

Figure 1. Gabor filters for 5 scales and 8 orientations.
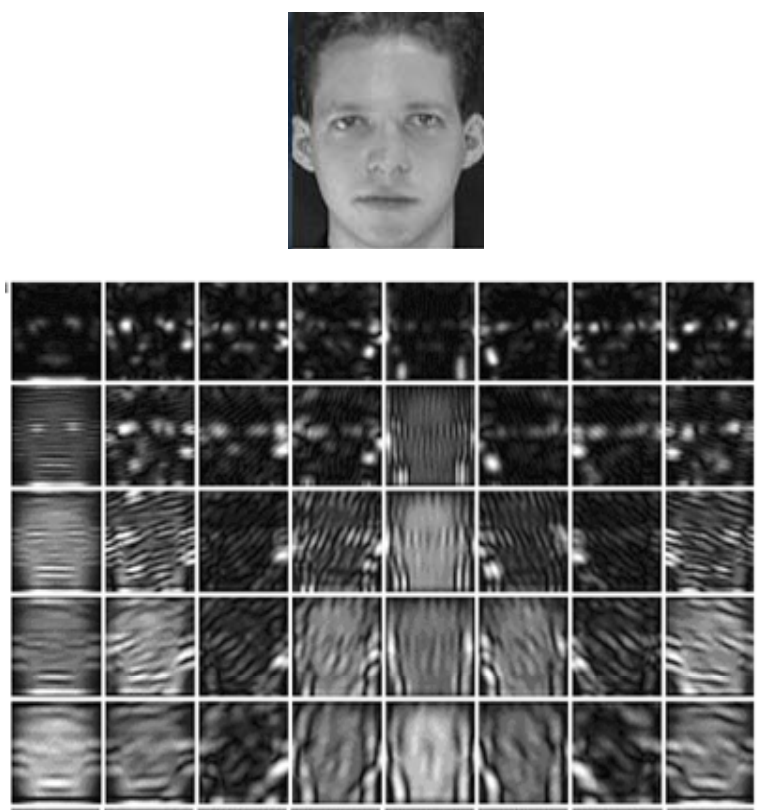

Figure 2. An input face image and the Gabor feature representation. subbands is denoted by LL, HL, LH, and HH. Each of these subbands can be thought of as a smaller version of the image representing different image properties.

\subsection{Discriminative Common Vector}

In order to obtain a low-dimensional feature representation with enhanced discrimination power it is proposed to construct discriminant features from the Gabor and wavelet coefficients using within-class scatter matrix method. A common vector for each individual class is obtained by removing all the features that are in the direction of the eigenvectors corresponding to the nonzero eigen values of within-class scatter matrix of all classes. The new set of vectors, called the discriminative common vectors, is used for recognition.

Let the training set be composed of $C$ classes, where each class contains $N$ samples. Let $x_{m}^{i}$ denotes the $m$ th sample from the $i$ th class. Within-class scatter matrix of the samples is constructed to obtain the feature vectors, which is defined as

$$
S_{w}=A A^{\mathrm{T}}
$$

where the matrix $\mathrm{A}$ is given by

$$
A=\left[x_{1}^{1}-\mu_{1}, \cdots, x_{N}^{1}-\mu_{1}, x_{1}^{2}-\mu_{2}, \cdots, x_{N}^{C}-\mu_{C}\right]
$$

where $x_{i}^{j}$ is the $i$-th sample of the class $j$ and $\mu_{j}$ is the mean of samples in the $j$ th class.

Let us define $Q=\left[\alpha_{1} \cdots \alpha_{r}\right]$, which is the set of orthonormal eigenvectors corresponding to the non-null eigenvalues of $S_{w}$ and $r$ is the dimension of $S_{w}$. The projection matrix can be expressed as $P=Q \bar{Q}$. Next choose an input sample and project it on the null space of $S_{w}$ in order to get the common vectors, defined as:

$$
x_{\mathrm{com}}^{i}=x_{m}^{i}+Q \bar{Q} x_{m}^{i}
$$

where $m=1 \cdots N$ samples and $i=1 \cdots C$ classes. Calculate the principal components of $S_{\text {com }}$ (the eigen-vectors $w_{k}$ ), which correspond to the non zero eigen-values as defined as:

$$
J\left(W_{\mathrm{opt}}\right)=\underset{w}{\arg \max }\left[W^{\mathrm{T}} S_{\mathrm{com}} W\right]
$$

where $S_{\text {com }}$ is computed as

$$
S_{\text {com }}=A_{\text {com }} A_{\text {com }}^{\mathrm{T}}
$$

where $A_{\text {com }}$ is given by

$$
A_{\text {com }}=\left[x_{\text {com }}^{1}-\mu_{\text {com }} \cdots x_{\text {com }}^{C}-\mu_{\text {com }}\right]
$$

The Feature Vector of Training set is calculated as

$$
\Omega_{i}=W^{\mathrm{T}} x_{m}^{i}
$$

Similarly, to recognize a test image $x_{\text {test }}$, the feature 

and Recognition Using RBF

vector of this test image is found by

$$
\Omega_{\text {test }}=W^{\mathrm{T}} x_{\text {test }}
$$

The above method is summarized as follows:

- Compute the nonzero eigenvalues and corresponding eigenvectors of $S_{w}$ using the matrix $A A^{\mathrm{T}}$, where A is computed using Equation (4).

- Choose an input sample from each class and project it onto the null space of $S_{w}$ to obtain the common vectors. Compute $x_{\text {com }}^{i}$ using Equation (5).

- Compute the eigenvectors $w_{k}$ of $S_{\text {com }}$, corresponding to the nonzero eigenvalues, by using the Equations (6) and (7).

- The feature vector for training set and test set is obtained using Equations (9) and (10) respectively.

\section{Recognition by Radial Basis Function Neural Network}

Radial Basis Function neural network (RBF) considered for recognition contains three layers: input, hidden and output. The number of nodes in the input layer corresponds to the dimension of extracted feature vector. The input neurons are normalized using the Equation (11), where $x_{i}$ is the $i$ th input vector. The normalized input values are fed to each of the neurons in the hidden layer. The basis function of the hidden layer neurons are considered to be Gaussian and the computed basis function output are passed to the output layer.

The hidden layer output is computed as

$$
\varphi_{j}(X)=\exp \left\{-\frac{\|x-\mu\|^{2}}{\sigma^{2}}\right\}
$$

where $X=\left(x_{1}, x_{2}, \cdots, x_{n}\right)^{\mathrm{T}}$ is the normalized input vector, $\mu$ is the center and $\sigma$ is the width.

The output layer output is computed as

$$
y_{i}=\sum_{j=1}^{k} w_{j i} \varphi_{j}(X)
$$

where $k$ is the number of hidden neurons, $w_{j i}$ are the weights connecting the hidden layer neuron $j$ and output layer neuron $i$. The weights are adjusted using the formula,

$$
w(t+1)=w(t)+\lambda\left(d_{i}-y_{j}\right) \varphi_{j}(X)
$$

where $\lambda$ is a positive learning rate parameter.

\subsection{Algorithm}

The training algorithm of Radial Basis Function Network is given as follows.

Step 1. Generate random number to initialize the weights of the RBF network.

Step 2. Enter the coefficients obtained from DCV.

Step 3. For each input pattern compute hidden layer output using the Equation (12).

Step 4. Compute the output layer output using the Equation (13).

Step 5. Find the error as the difference between desired and actual output obtained.

Step 6. Adjust the hidden layer weights according to Equation (14).

Step 7. Find output of the output layer.

Step 8. Compute sum of squared error of the network.

Step 9. Repeat steps 3-8 for all input patterns.

Step 10. Repeat steps 3-9 until the acceptable minimum error level is reached.

The proposed work is shown in Figure 3. The Entire work is summarized as follows.

- For each training set face images, perform steps 2-5,

- Compute the Gabor filters using the Equation (1).

- Find the Gabor representation of the face images by convolving the input face image and the Gabor filters using the Equation (2).

- Reduce the dimension of the Gabor features by applying the wavelet transformation.

- Compute the DCV Coefficients for the computed wavelet coefficients of step 4 using the within class scatter matrix method.

- Train the RBF network for recognizing DCV coefficients using the algorithm in Section 3.1.

- Repeat the steps 2-5 for the test set face images.

- Classify the DCV coefficients of test image using the trained RBF network.

\section{Results and Discussions}

The proposed system is tested using the face databases such as ORL, The Japanese Female Facial Expression (JAFFE) and Essex Face database.

The ORL face data base contains 40 faces and each face has 10 different facial views representing various expressions, small occlusion by glasses, different scale and orientations. Hence, there are 400 face images in the database and each 100 images of 20 persons are used for training and another 100 images of the 20 persons are used for testing. The resolution of all the images is $112 \times$ 92.

Gabor wavelet representations such as the real part and

$$
x_{i}=2\left(x_{i}-\left(\min \left(x_{i}\right) * \text { unitvec }\right)\right) /\left(\max \left(x_{i}\right)-\min \left(x_{i}\right)\right)-\text { unitvec }
$$




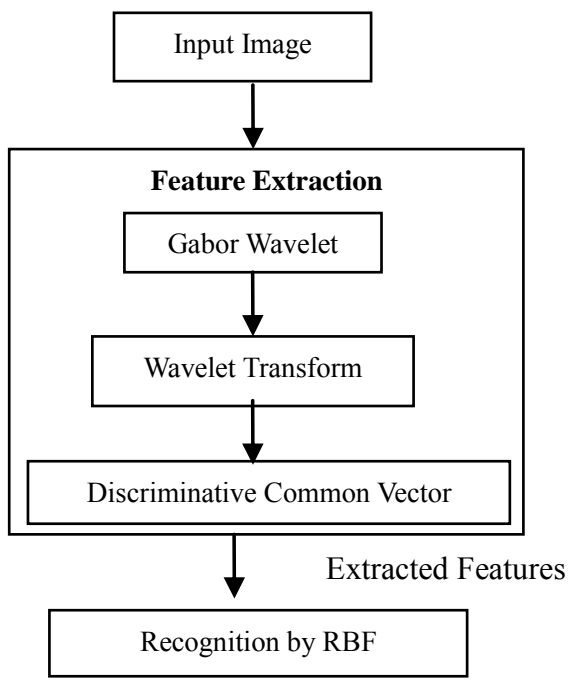

Figure 3. Proposed recognition system.

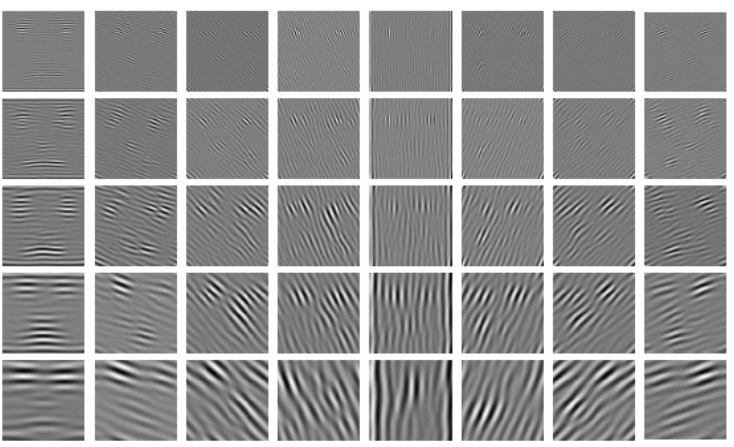

Figure 4. Real part of Gabor wavelet representation.
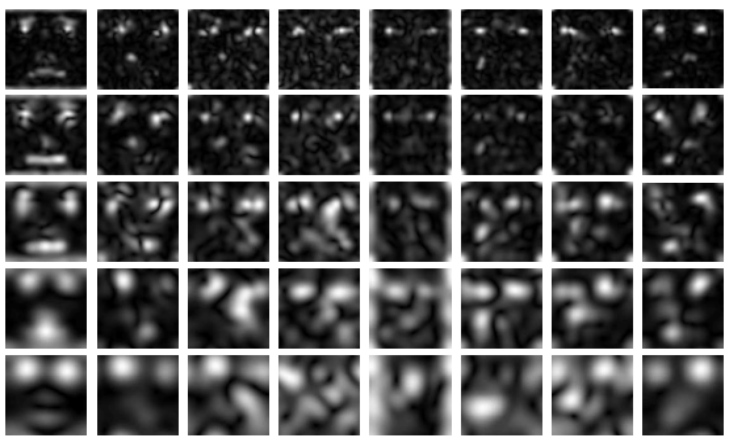

Figure 5. Magnitude part of Gabor wavelet representation.

the magnitude part of a sample image are shown in Figures 4 and 5 respectively. Different wavelets namely Haar, Symlet, Daubechies and Coiflets are used during wavelet transformation. The original, resultant large dimensional Gabor images and the reduced gabor images after applying wavelet transformation along with their sizes are shown in Figures 6(a)-(c). The recognition rates along with training time and epoch obtained for different wavelets are shown in Table 1. The recognition rates for different wavelets namely Haar, Sym4, Sym8, Db4, Db6, Coif2, Coif4 are 98.7\%, 97.0\%, 96.67\%, $97.33 \%, 96.33 \%, 97.00 \%$ and $96.37 \%$ respectively.

The Japanese Female Facial Expression contains 213 images of 7 facial expressions ( 6 basic facial expressions +1 neutral) posed by 10 Japanese female models. Each image has been rated on 6 emotion adjectives by 60 Japanese subjects. The actual dimension of the image is $256 \times 256$. The epoch, training time and recognition rate obtained for JAFFE Database are listed in Table 2. The highest recognition rate is $98.88 \%$ for Haar wavelet and $96.7 \%$ for Sym8. Lowest training time 18.01 seconds is used when Sym8 wavelet is applied.

The Essex Face database is having faces of more than
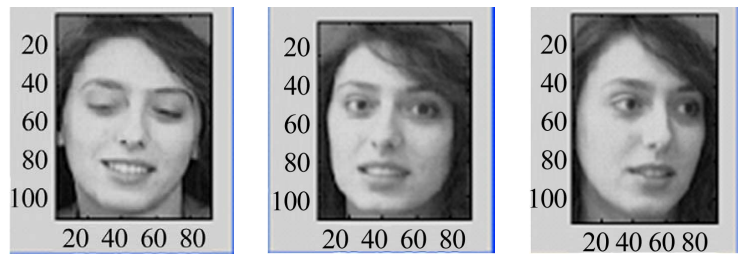

(a)
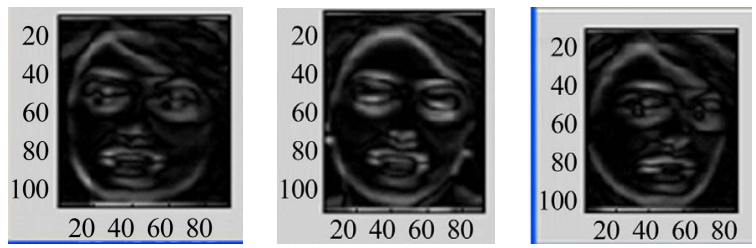

(b)
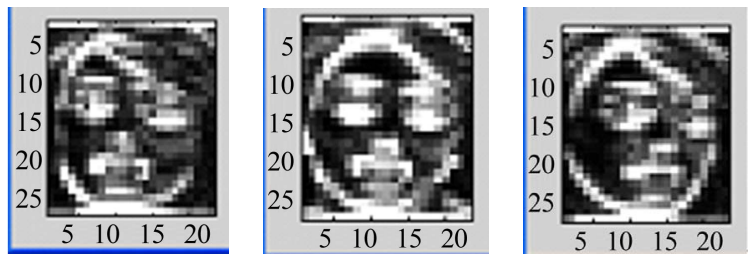

(c)

Figure 6. (a) Original image with size $112 \times 92$; (b) Gabor face representation with size $112 \times 92$; (c) Gabor + Wavelet representation with size $28 \times 23$.

Table 1. Results of ORL Database.

\begin{tabular}{cccc}
\hline $\begin{array}{c}\text { Wavelet } \\
\text { Name }\end{array}$ & $\begin{array}{c}\text { Recognition } \\
\text { Rate (\%) }\end{array}$ & $\begin{array}{c}\text { Training Time } \\
\text { in Seconds }\end{array}$ & Epoch \\
\hline Haar & 98.7 & 9.28 & 324 \\
Sym4 & 97.0 & 9.6 & 363 \\
Sym8 & 96.67 & 10.12 & 352 \\
Db4 & 97.33 & 9.79 & 339 \\
Db6 & 96.33 & 10.38 & 361 \\
Coif2 & 97.00 & 10.48 & 337 \\
Coif4 & 96.37 & 11.97 & 348 \\
\hline
\end{tabular}


150 male and female with 20 images per individual of University of Essex, UK. The various results obtained on Essex face database are presented in Table 3.

Two more feature extraction methods are carried out in this work for comparative purpose. In the first method, the features are extracted using the wavelet transformation and DCV. In the second method, the Gabor representation for the face obtained are averaged then applied to wavelet transformation and then to DCV method. These two methods are tested on the ORL, JAFFE and Essex face databases using different types of wavelets and the results are shown in Table 4.

When comparing the previous works, the performance of the proposed work is better than the other methods. The recognition rate of hybrid method of wavelet, DCV and RBF (WDR) is $97.3 \%$ for ORL dataset, where as the wavelet face along with Neural Network yields the recognition rate of $93.5 \%$. After combining the Gabor feature extraction with the WDR, the proposed method Gabor, wavelet, DCV and RBF (GWDR) is yielded the improved recognition rate as $98.7 \%$ for ORL, $98.88 \%$ for JAFFE and $98.33 \%$ for ESSEX database.

The recognition rates of these methods are less when compared with the proposed method which is a hybrid of Gabor, wavelet transformation and DCV method. When comparing these two methods, wavelet and DCV based feature extraction yields better results than the hybrid

Table 2. Results of JAFFE database.

\begin{tabular}{cccc}
\hline $\begin{array}{c}\text { Wavelet } \\
\text { Name }\end{array}$ & $\begin{array}{c}\text { Recognition } \\
\text { Rate (\%) }\end{array}$ & $\begin{array}{c}\text { Training Time } \\
\text { in Seconds }\end{array}$ & Epoch \\
\hline Haar & 98.88 & 19.44 & 380 \\
Sym4 & 98.7 & 18.22 & 344 \\
Sym8 & 96.7 & 18.01 & 334 \\
Db4 & 98 & 18.54 & 349 \\
Db6 & 97.33 & 19.02 & 353 \\
Coif2 & 98 & 19.31 & 359 \\
Coif4 & 97.7 & 18.52 & 339 \\
\hline
\end{tabular}

Table 3. Results of ESSEX database.

\begin{tabular}{cccc}
\hline $\begin{array}{c}\text { Wavelet } \\
\text { Name }\end{array}$ & $\begin{array}{c}\text { Recognition } \\
\text { Rate (\%) }\end{array}$ & $\begin{array}{c}\text { Training Time } \\
\text { in Seconds }\end{array}$ & Epoch \\
\hline Haar & 98.33 & 19.44 & 380 \\
Sym4 & 98.4 & 18.22 & 324 \\
Sym8 & 96.7 & 18.01 & 311 \\
Db4 & 98.33 & 18.54 & 349 \\
Db6 & 97 & 19.02 & 353 \\
Coif2 & 98.11 & 19.31 & 364 \\
Coif4 & 97.33 & 18.5 & 337 \\
\hline
\end{tabular}

method of averaged Gabor, wavelet transformation and DCV. The recognition rate obtained using the three face databases on applying other methods are shown in Table 5. The recognition rates of the ORL, JAFFE and Essex

Table 4. Recognition rates.

\begin{tabular}{ccccccc}
\hline \multirow{2}{*}{$\begin{array}{c}\text { Wavelet } \\
\text { Name }\end{array}$} & \multicolumn{3}{c}{ Wavelet + DCV + RBF } & \multicolumn{2}{r}{$\begin{array}{r}\text { Averaged Gabor + Wavelet } \\
\text { + DCV + RBF }\end{array}$} \\
\cline { 2 - 7 } & \multicolumn{2}{c}{ Recognition Rate (\%) } & \multicolumn{3}{c}{ Recognition Rate (\%) } \\
\cline { 2 - 7 } & ORL & JAFFE & ESSEX & ORL & JAFFE & ESSEX \\
\hline Haar & 97.3 & 97.3 & 96.8 & 96.33 & 90.33 & 91.33 \\
Sym4 & 96.7 & 97 & 95 & 96 & 90 & 91 \\
Sym8 & 95.0 & 95.7 & 95.4 & 96 & 90 & 89 \\
Db4 & 96.0 & 96.7 & 95.33 & 94.33 & 89.33 & 92 \\
Db6 & 95.4 & 96.4 & 94.0 & 94.0 & 88 & 90.37 \\
Coif2 & 96.17 & 96 & 95.0 & 96.0 & 88.4 & 92 \\
Coif4 & 95.0 & 95.3 & 94.67 & 96.33 & 89.1 & 92.7 \\
\hline
\end{tabular}

Table 5. Comparison of recognition rates.

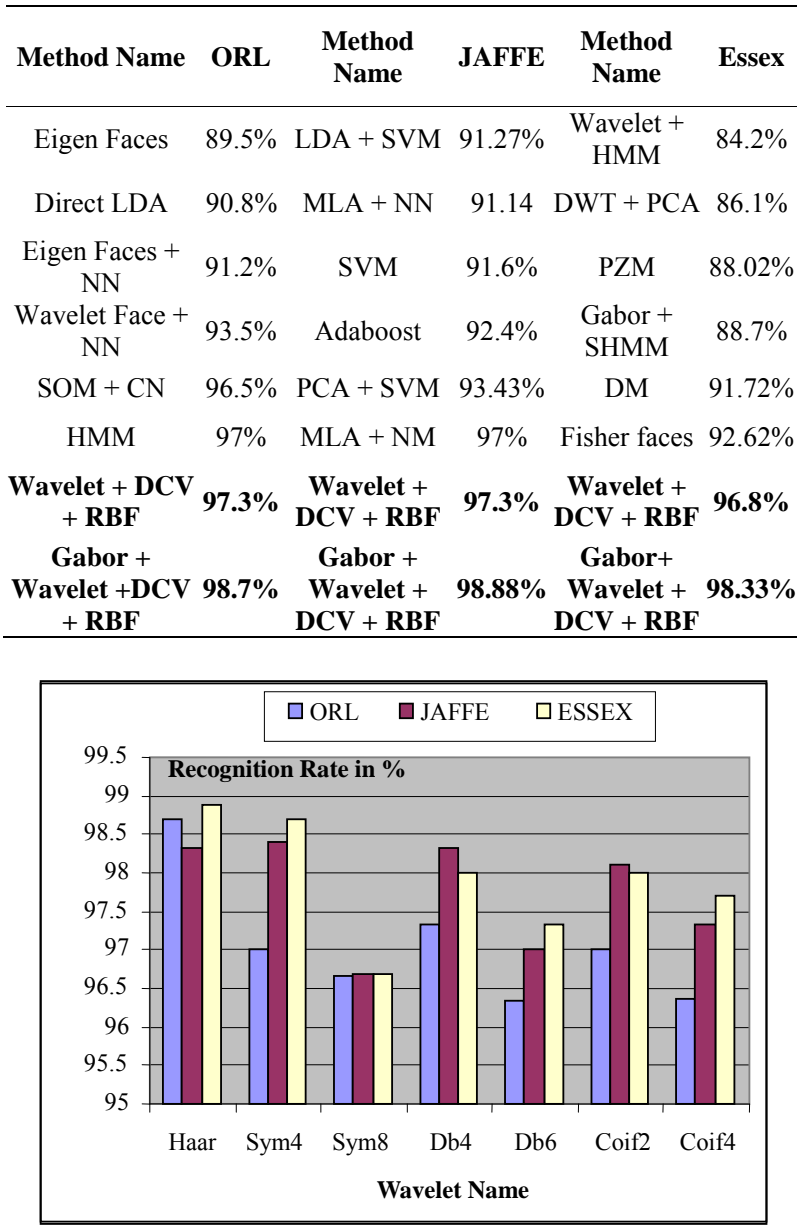

Figure 7. Comparison of recognition rates of three databases for different wavelets. 
face databases for different types of wavelets are shown in Figure 7.

\section{Conclusion}

The performance of a face recognition system depends not only on the classifier, but also on the face representation. A face recognition system is devised with effective face representation by the combined approach of Gabor filter, wavelet transformation and discriminative common vectors and recognition by radial basis function (RBF) neural network. The proposed system reduces the number of features, minimizes the computational complexity and yielded the better recofgnition rates for ORL database, JAFFE face database and ESSEX database. The recognition performance of the classification is improved due to the hybrid technique used in the feature extraction stage which provides necessary information for classification.

\section{REFERENCES}

[1] C. J. Liu and H. Wechsler, "Gabor Feature Based Classification Using the Enhanced Fisher Linear Discriminant Model for Face Recognition," IEEE Transactions on Image Processing, Vol. 11, No. 4, 2002, pp. 467-476.

[2] M. Turk and A. Pentland, "Eigenfaces for Recognition," Cognitive Neuroscience, Vol. 3, No. 1, 1991, pp. 71-86. doi:10.1162/jocn.1991.3.1.71

[3] P. Belhumeur, J. Hespanha and D. Kriegman, "Eigenfaces vs Fisher Faces: Recognition Using Class Specific Linear Projection," IEEE Transactions on Pattern Analysis and Machine Intelligence, Vol. 20, No. 7, 1997, pp. 711-720. doi: $10.1109 / 34.598228$

[4] M. Bartlett, J. Movellan and T. Sejnowski, "Face Recognition by Independent Component Analysis," IEEE Transactions on Neural Networks, Vol. 13, No. 6, 2002, pp. 1450-1464. doi:10.1109/TNN.2002.804287

[5] Y.-C. Lee and C.-H. Chen, "Feature Extraction for Face Recognition Based on Gabor Filters and Two-Dimensional Locality Preserving Projections," Proceedings of the 5th IEEE Conference on Intelligent Information Hiding and Multimedia Signal Processing, Kyoto, 12-14 September 2009, pp. 106-109.

[6] L. Shen and L. Bai, "Face Recognition Based on Gabor Features Using Kernel Methods," Proceedings of the 6th IEEE Conference on Face and Gesture Recognition, Seoul, 17-19 May 2004, pp. 170-175. doi:10.1109/AFGR.2004.1301526

[7] S. F. Xie, S. G. Shan, X. L. Chen and J. Chen, "Fusing Local Patterns of Gabor Magnitude and Phase for Face Recognition," IEEE Transactions on Image Processing, Vol. 19, No. 5, 2010, pp. 1349-1361. doi:10.1109/TIP.2010.2041397

[8] X. H. Li, K.-M. Lam, L. S. Shen and J. L. Zhou, "Face Detection Using Simplified Gabor Features and Hierar- chical Regions in a Cascade of Classifiers," Pattern Recognition Letters, Vol. 30, No. 8, 2009, pp. 717-728. doi:10.1016/j.patrec.2009.03.006

[9] T. P. Weldon, W. E. Higgins and D. F. Dunn, "Efficient Gabor Filter Design for Texture Segmentation," Pattern Recognition, Vol. 29, No. 12, 1996, pp. 2005-2015. doi:10.1016/S0031-3203(96)00047-7

[10] Y. Hamamoto, S. Uchimura, M. Watanabe, T. Yasuda, Y. Mitani and S.Tomita, "A Gabor Filter-Based Method for Recognizing Handwritten Numerals," Pattern Recognition, Vol. 31, No. 4, 1998, pp. 395-400. doi:10.1016/S0031-3203(97)00057-5

[11] C. J. Lee and S. D. Wang, "Fingerprint Feature Extraction Using Gabor Filters," Electronics Letters, Vol. 35, No. 4, 1999, pp. 288-290. doi:10.1049/el:19990213

[12] C. J. Liu and H. Wechsler, "Independent Component Analysis of Gabor Features for Face Recognition," IEEE Transactions on Neural Networks, Vol. 14, No. 4, 2003, pp. 919-928.

[13] A. Kar, D. Bhattacharjee, D. K. Basu, M. Nasipuri and M. Kundu, "High Performance Human Face Recognition Using Independent High Intensity Gabor Wavelet Responses: A Statistical Approach," International Journal of Computer Science \& Emerging Technologies, Vol. 2, No, 1, 2011, pp. 178-187.

[14] L. L. Shen, L. Bai and M. Fairhurst, "Gabor Wavelets and General Discriminant Analysis for Face Identification and Verification," Image and Vision Computing, Vol. 25, No. 5, 2007, pp. 553-563. doi:10.1016/j.imavis.2006.05.002

[15] W.-P. Choi, S.-H. Tse, K.-W. Wong and K.-M. Lam, "Simplified Gabor Wavelets for Human Face Recognition," Pattern Recognition, Vol. 41, No. 3, 2008, pp. 1186-1199. doi:10.1016/j.patcog.2007.07.025

[16] H. Cevikalp, M. Neamtu, M. Wilkes and A. Barkana, "Discriminative Common Vectors for Face Recognition," IEEE Transactions on Pattern Analysis and Machine Intelligence, Vol. 27, No. 1, 2005, pp. 4-13. doi:10.1109/TPAMI.2005.9

[17] L. Wiskott, J. M. Fellous, N. Kuiger and C. Vonder Malsburg, "Face Recognition by Elastic Bunch Graph Matching," IEEE Transactions on Pattern Analysis and Machine Intelligence, Vol. 9, No. 7, 1997, pp. 775-779. doi:10.1109/34.598235

[18] S. Lahmiri and M. Boukadoum, "Hybrid Discrete Wavelet Transform and Gabor Filter Banks Processing for Mammogram Features Extraction," Proceedings of IEEE 9th International Conference New Circuits and Systems Conference (NEWCAS), Bordeaux, 26-29 June 2011.

[19] T. M. Mitchell, "Machine Learning," China Machine Press, Beijing, 2003.

[20] X.-Y. Jing, Y.-F. Yao, J.-Y. Yang and D. Zhang “A Novel Face Recognition Approach Based on Kernel Discriminative Common Vectors (KDCV) Feature Extraction and RBF Neural Network," Neurocomputing, Vol. 71, No. 13-15, 2008, pp. 3044-3048. doi:10.1016/j.neucom.2007.08.027

[21] M. Balasubramanian, S. Palanivel and V. Ramalingam, 

and Recognition Using RBF

"Real Time Face and Mouth Recognition Using Radial Basis Function Neural Networks," Expert Systems with Applications, Vol. 36, No. 3, 2009, pp. 6879-6888. doi:10.1016/j.eswa.2008.08.001

[22] J. G. Daugman, "Complete Discrete 2D Gabor Transforms by Neural Networks for Image-Analysis and Compression," IEEE Transactions on Acoustic Speech Signal
Process, Vol. 36, No. 7, 1988, pp. 1169-1179. doi:10.1109/29.1644

[23] V. Kyrki, J. K. Kamarainen and K. Kalviainen, "Simple Gabor Feature Space for Invariant Object Recognition," Pattern Recognition Letters, Vol. 25, No. 3, 2004, pp. 311-318. doi:10.1016/j.patrec.2003.10.008 